\title{
Deterministic approximation of stochastic spatially explicit model of actin-myosin interaction in discrete filament lattice
}

\author{
Artem M. Mishchenko, Olga I. Dotsenko and Galina V. Taradina \\ Biophysical Department, "Vasyl' Stus” Donetsk National University, Vinnitsa, Ukraine
}

\begin{abstract}
One of commonly used approaches of biophysical modeling of muscle contractile apparatus is spatially explicit discrete lattice models in Monte Carlo simulation. Such models allow to reproduce structural features and actin-myosin interaction in the muscle contractile system more accurately. Limitation of such models is their low computational efficiency and stochasticity under certain circumstances. This work introduces deterministic approximation of stochastic model that considers a pair of rigid contractile filaments interaction. Approximation background is discreetness of spacing between cross-bridges and binding sites. Due to this property cross-bridges can be divided into discrete groups with the same strain, and considered statistically using the set of ordinary differential equations. Deterministic model is more computationally efficient, operates with average values. Within the given approach isotonic contraction was simulated. A comparison with Monte Carlo simulation demonstrates that approximation reproduces results for stochastic model with large number of cross-bridges. Also within the deterministic model a mechanism and essential conditions for oscillations appearance in isotonic transient response, relations of their parameters with geometrical ones of filaments lattice were examined, theoretical and experimental results were compared. The proposed approach can also be applied to approximation of continuous Huxley-based models solutions. Advantage over existing numerical methods is their greater numerical stability.
\end{abstract}

Key words: Sarcomere lattice geometry - Molecular mechanism - Spatially explicit model Deterministic approximation - Stepwise contraction

\section{Introduction}

Molecular mechanism of the functioning of such complicated system as muscle cannot be obtained only by studying the properties of individual isolated components of this system, due to it emergent character. In particular, muscle sarcomere architecture plays an important role in this mechanism. Work performed by a muscle is the result of the interaction between cross bridges of thick filaments and actin of thin filaments. This interaction depends on their mutual position, which in turn is determined by the structure of myofilaments

Electronic supplementary material. The online version of this article (doi: 10.4149/gpb_2018005) contains supplementary material which is available to authorized users.

Correspondence to: Artem Mikhailovich Mishchenko, 4 Akademika Yanhelia str., Vinnitsa 21007, Ukraine

E-mail: a.mishchenko@donnu.edu.ua and their arrangement in sarcomere lattice. Physiological significance of a sarcomere structure is highlighted by the fact that the degree of its order has changed in the course of evolution (Squire et al. 2005, 2008; Iwamoto et al. 2006) and that muscles with different sarcomere architecture differ in their functional characteristics (Squire et al. 2008; Iwamoto 2011). The mechanism that links features of sarcomere lattice geometry to its mechanical characteristic is still poorly explored.

One of the currently widely used approaches to study molecular mechanism of muscle contraction is computer simulation. In many models of muscle contraction the approach that originates from classical Huxley model (Huxley 1957) is applied. In this approach, the internal state of sarcomere is described at the level of cross-bridges distributions along reaction coordinate, which depends on relative distance between cross-bridges and actin binding site. It is assumed that this distance is a continuous quantity with a uniform distribution among all cross-bridges. Time 
evolution of cross-bridges distributions is described using partial differential equations (PDE). A disadvantage of this approach is that it doesn't explicitly take into account sarcomere lattice geometry.

Another, more recent type of the models is spatially explicit stochastic models (Daniel et al. 1998; Campbell 2006). In this approach, the contractile system state is specified more detailed to the level of mechano-chemical state of individual cross-bridges. Cross-bridge state transitions are modeled with Monte Carlo methods. While keeping information about the features of sarcomere lattice geometry this approach more realistically reproduces interaction between cross-bridges and binding sites. In contrast to Huxley-based models, the distance between cross-bridges and binding sites is discrete quantity.

Theoretical studies performed in the course of the approach that use spatially detailed models prove influence of the structure on kinetics of actomyosin interactions, ATP utilization, muscle force production, thin-filament $\mathrm{Ca}^{2+}$ activation (Duke 1999; Tanner et al. 2007; Smith et al. 2008). Behavior of cross-bridges ensemble demonstrates complex collective effects absent in spatially continuous models (Duke 1999; Smith et al. 2008). Fluctuations, which occur in this model due to stochasticity of the cross-bridge kinetic transitions, also can be relevant to functioning of the contractile system. Capability to simulate within this approach the contractile system, which is composed of limited number of filaments, is actual due to experimental studies of such systems (Liu et al. 2004; Suzuki et al. 2005; Placais et al. 2009).

However, this approach also has its disadvantages: models, created within this approach, are computationally demanding. Using individual realizations of the stochastic dynamical process it can be difficult to obtain its general properties, define its dependence on parameters. It is not always possible to separate these general properties from occasional fluctuations by averaging individual realizations. The fact that the model operates with random realizations may cause problems in the case of analysis of model results, its parametric identification or its sensitivity analysis.

So, it is advantageous to construct the model, allowing the acceleration of the simulation and operating with average values, as well as taking into account information on the structure of the sarcomere lattice.

As the results of experimental researches suggest, muscle contractile apparatus can operate in oscillatory mode, the nature of this phenomenon thought to be tightly related to sarcomere structure. Isolated muscle fibers from the frog exhibit damped length oscillations in the isotonic transient response to a force step (Edman et al. 2001). When myofibril from rabbit skeletal or cardiac muscles or from insects flying muscles was ramp-released or ramp-stretched by motor, their individual sarcomere length changes in stepwise fashion (Yakovenko et al. 2002; Nagornyak et al. 2004; Pollack et al.
2005). Similarly, at the level of the single pair of thick and thin filaments, sliding of thin filament against elastic load follows a stepwise trajectory (Liu et al. 2004). It is considered that such oscillatory behavior reflects synchronous activity of the cross-bridges. This synchronization may be based on restrictions imposed by filament lattice on myosin and actin interaction. Indirectly, this is indicated by quantitative relations between the parameters of oscillatory dynamics and geometrical parameters of the lattice. Thus, in (Edman et al. 2001) peak-to-peak amplitude of the muscle fiber length oscillation was equal to $2.7 \mathrm{~nm}$. And in researches (Yakovenko et al. 2002; Liu et al. 2004; Nagornyak et al. 2004; Pollack et al. 2005) the height of steps in sliding trajectory of sarcomeres or pair of filaments was equal to integer multiple of $2.7 \mathrm{~nm}$. On the other hand, the value of $2.7 \mathrm{~nm}$ is equal to the monomeric repeat along the actin filament (Pollack et al. 2005).

Oscillatory sliding was also theoretically proved in the number of theoretical studies that applied the models with discrete lattice (Duke 1999; Smith et al. 2008). Those studies imply that such collective dynamics results from interaction between individual cross-bridges mediated by common thin filaments with which they interact. If one-cross bridge shifts a thin filament, it will change interactions of the other crossbridges with it. It was suggested that the oscillatory dynamics retrieved in the models is analogous to experimental (Duke 1999; Vilfan et al. 2003; Smith et al. 2008). However, the step value in those works has not coincided with experimental one and quantitative relation between the step value and model parameters was not provided either.

In this research the approach for deterministic approximation of the stochastic spatially explicit model of a pair of rigid contractile filaments interaction was developed. The starting point for approximation is discreetness of crossbridges strain that also determines their chemical properties. As a result, all cross-bridges can be divided into finite number of groups by their strain (chemical properties). If each group has a great number of cross-bridges, then instead of individual cross-bridge state simulation it is possible to statistically simulate the whole group state via relevant kinetic equations. Such approximation preserves information about filament lattice geometry, defined by the set of ordinary differential equations (ODEs), and in contrast to stochastic model it operates with average values.

To verify approximation adequacy, we compared the results of deterministic ODE model with Monte Carlo simulation. Also within ODE model we studied in detail a mechanism and essential conditions for oscillations appearance under isotonic contraction, linked their spatial period with geometrical parameters of filaments lattice and compared theoretical and experimental results.

The suggested approach can also be used for approximation of PDE models solutions based on Huxley formalism. So, we also validated the adequacy of such approximation; 


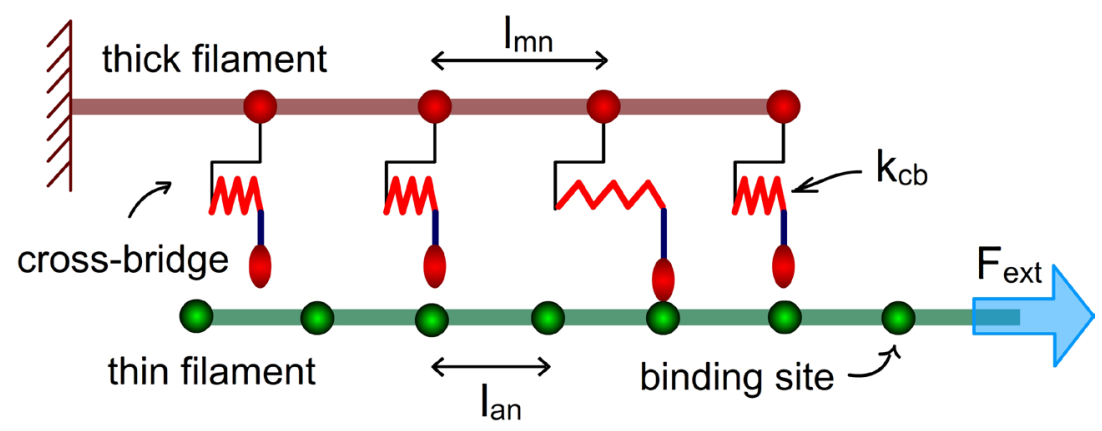

Figure 1. Contractile system composed of a single pair of inextensible thick and thin filaments. compare numerical stability of the given method with existing numerical methods for PDE solving.

\section{Material and Methods}

Spatially explicit stochastic model of a single pair of contractile filaments

We were looking for an approximation of stochastic spatially explicit model with a single pair of non-extensible thick and thin filaments (Fig. 1). There are $N$ cross-bridges on think filament with spacing $l_{m n}$ between adjacent, which can interact with binding sites of thin filament. It is assumed that at the same time only one of the two cross-bridge heads can bind to actin. Thin filament has infinite number of binding sites with spacing $l_{a n}$ between adjacent. The thick filament is fixed, while thin filament can move in longitudinal direction when subjected to the forces generated by bound cross-bridges and external force $F_{\text {ext }}$. The force of bound cross-bridges arises from deformation of their elastic element with spring constant $k_{c b}$.

In general, cross-bridges cycle can include a number of free and bound states. Further we will consider kinetic crossbridge cycle that includes three states:

$1 \underset{k_{21}}{\stackrel{k_{12}}{\rightleftarrows}} 2 \underset{k_{32}}{\stackrel{k_{23}}{\rightleftarrows}} 3 \stackrel{k_{31}}{\longrightarrow} 1$

where numbers indicate different chemical states: state 1 is detached, states 2 and 3 are bound; $k_{i j}$, rate for transition $i \rightarrow j$. Transitions $1 \rightarrow 2$ and $2 \rightarrow 3$ may be accompanied by conformational changes in the myosin head, which increase the cross-bridge elastic element distortion by values $d_{1}$ and $d_{2}$ respectively. Transition $3 \rightarrow 1$ is accompanied by recovery stroke with value of $-\Sigma^{2}{ }_{i=1} d_{i}$. Chemical transition of individual cross-bridge is stochastic event and it is simulated by Monte Carlo method (Daniel et al. 1998).

It is supposed that in general case transition rates $k_{i j}$ are functions of cross-bridge distortion, which in turn depends on relative axial position between cross-bridge and binding site with which it interacts. We assume that thick filament is fully overlapped by thin filament. Each cross-bridge is characterized by relative axial distance from its head equilibrium position in unbound state to the closest actin binding site on the right. Due to the periodicity of the cross-bridges and actin binding sites locations this distance will be a discrete quantity, and under the given mutual arrangement of thin and thick filaments, it will take values from discrete set $\Delta=\left\{\Delta^{1}, \ldots \Delta^{\mathrm{k}}, \ldots \Delta^{\mathrm{M}}\right\}$, where $\Delta^{\mathrm{k}+1}>\Delta^{\mathrm{k}}$. Size $M$ and values of the set $\Delta$ depend on cross-bridges and binding sites periodicity $\left(l_{m n}\right.$ and $\left.l_{a n}\right)$, as well as on number of cross-bridges $N$.

Further, we find the properties for the discrete set $\Delta$ in case of the infinitely long thick filament. Having chosen $i$-th cross-bridge (Fig. 2), we can find a cross-bridge with number of $i+T_{c b}$ along thick filament which will have the same distance to the closest binding site on the right as $i$-th one. The distances to the closest right-handed binding site of cross-bridges from $i$-th to $i+T_{c b}-1$, make a pattern of unique values. Beginning with $i+T_{c b}$-th cross-bridge, this pattern will repeat. Sorted values of this pattern will constitute a set $\Delta$. In this case, the size of the set $\Delta$ will be $M=T_{c b}$. The value $T_{c b}$ is related to geometrical parameters of lattice as follows:

$l_{m n} T_{c b}=l_{a n} T_{b s}$

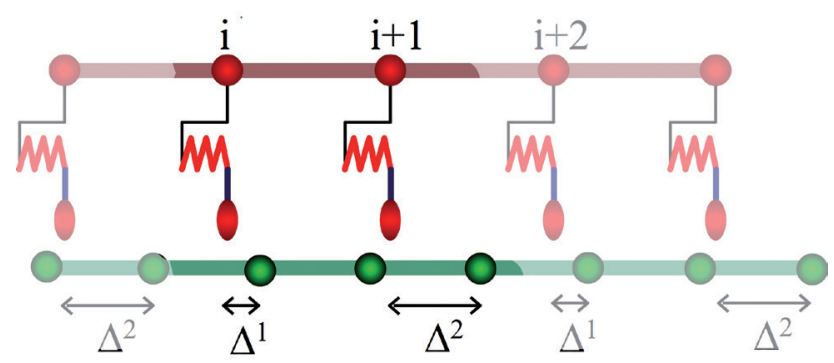

Figure 2. Example of lattice of a single pair of thick and thin filaments for which the set $\Delta$ consists of two values $\left\{\Delta^{1}, \Delta^{2}\right\}$. Crossbridges with numbers of $i+T_{c b} k$ and $i+T_{c b} k+1$, where $T_{c b}=2$ and $k$ is integer, the distances to the closest binding site on the right are equal to $\Delta^{1}$ and $\Delta^{2}$ respectively. 

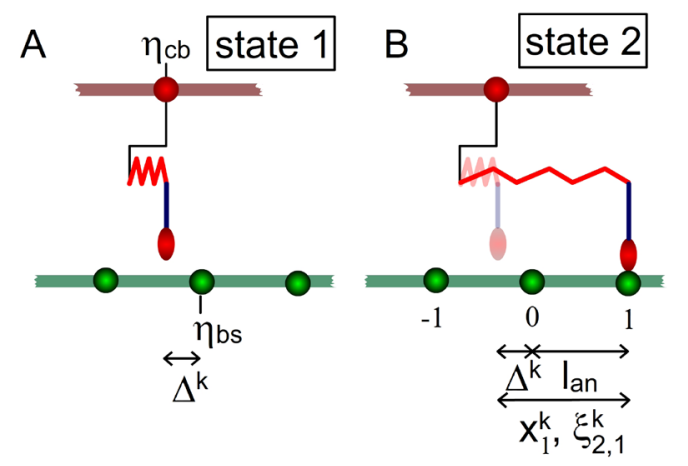

Figure 3. Relationship between values of $\Delta^{\mathrm{k}}, x^{k}$ and $\xi_{i, r}^{k}$ for the cross-bridge in different chemical states. Figures illustrate the same cross-bridge in different states for the same mutual offset between thick and thin filaments. A. In unbound state 1 , the distance between myosin head and the closest binding site on the right is equal to $\Delta^{k}$. B. Under the actin binding sites their relative numbers are shown. The cross-bridge is connected with 1-th binding site, then $x_{r}^{k}=\Delta^{k}+l_{a n}$. In the given example it is supposed that transition $1 \rightarrow 2$ is not accompanied by conformational changes of cross-bridge $\left(d_{1}=0\right)$ so the value of cross-bridge elastic element distortion $\xi_{2,1}^{k}$ is equal to $x^{k}{ }_{1}$. C. If transition $2 \rightarrow 3$ is accompanied by structural changes of cross-bridge which performs the power stroke of size $d_{2}$ then under transition to the state 3 cross-bridge elastic element distortion will increase by $d_{2}: \xi_{3,1}^{k}=x^{k}{ }_{1}+d_{2}$.

where $T_{c b}$ and $T_{b s}$ are some unknown integers and they should be the least among possible. Distance $s$ between any two adjacent elements of $\Delta$ will be:

$$
s \equiv \Delta^{k+1}-\Delta^{k}=l_{m n} / T_{b s}=l_{a n} / T_{c b}
$$

and elements of the set $\Delta$ will have the following values:

$$
\Delta^{k}=k s+C, \quad k=1, \ldots, T_{c b}, \quad \Delta^{k} \in\left[0, l_{a n}\right)
$$

where the value of the constant $C$ depends on current mutual offset of thick and thin filaments.

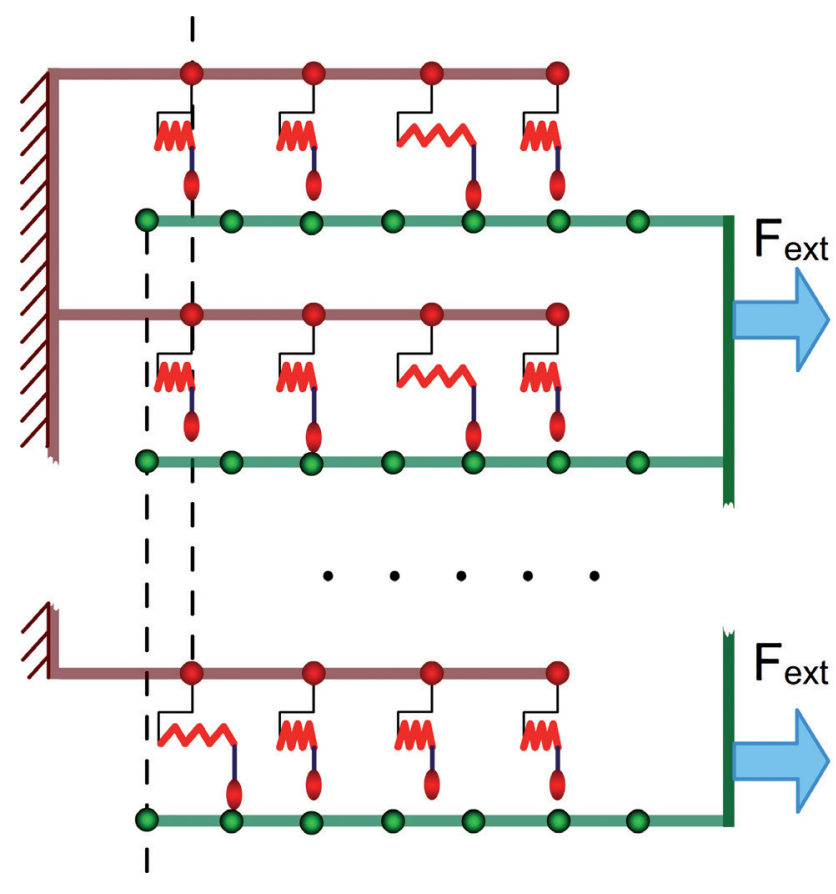

Figure 4. Contractile system composed from numerous pairs of inextensible thick and thin filaments connected in parallel.
For the thick filament of finite length, a number of crossbridges $N$ in general case will not be equal to $T_{c b}$. If $N<T_{c b}$ then $M=N$ and current set $\Delta$ does not include all the values from the set (Eq. 4). Differences $\Delta^{k+1}-\Delta^{k}$ between adjacent elements of the set $\Delta$ will be multiple of $s$. If $N>T_{c b}$ and $N$ is not multiple of $T_{c b}$ then $M=T_{c b}$ and the set $\Delta$ will include all elements (Eq. 4 ) but their distribution between $N$ crossbridges will be non-uniform.

The distance from cross-bridge to binding site with relative number $r$, with which it interacts (see Fig. 3), is equal:

$x_{r}^{k}=\Delta^{k}+r l_{a n}, \quad r=-m, \ldots, n$

Binding sites are numbered so that the closest one on the right has its number $r=0$, and on the left $r=-1$ etc. (Fig. 3).

The cross-bridge distortion is expressed by $x^{k}{ }_{r}$ in the following way (see Fig. 3):

$\xi_{i, r}^{k}\left(x_{r}^{k}\right)=\left\{\begin{array}{cc}x_{r}^{k}=\Delta^{k}+r l_{a n} & i=1 \\ x_{r}^{k}+\sum_{k=1}^{i-1} d_{k}=\Delta^{k}+r l_{a n}+\sum_{k=1}^{i-1} d_{k} & i=2,3\end{array}\right.$

where $\xi_{i, r}^{k}$ is distortion of the cross-bridge in $i$-th chemical state. For unbound state $\xi_{i, r}^{k}$ is distortion which must be taken by cross-bridge elastic element due to thermal fluctuations for successful binding with $r$-th binding site.

\section{Deterministic approximation}

In the system with a single pair of thick and thin filaments (Fig. 1) stochasticity can be eliminated by combining a number of such pairs subsequently (Fig. 4). State of the combined system will represent an average of all possible stochastic realizations of the single pair filaments system state at the given moment of time. Dynamics of the combined system can be modeled using the system of ODEs. 
In contractile system composed of large number of thickthin filament pairs every thick filament is inflexibly linked to each other and any two cross-bridges with the same number, located on different thick filaments, have the same axial position (Fig. 4). The same is true for thin filaments and binding sites on them. Because the thick and thin filaments and links between them are rigid, at mutual sliding of thick and thin filaments, the distances $\Delta^{k}$ of all cross-bridges will change in the same way. That is, cross-bridges of all pairs of contractile filaments, at any given moment, will have the same sets of $\Delta$. All cross-bridges of the combined system can be divided into finite number of groups $(M)$ on the basis of their distance to the closest binding site on the right. Within each group, all cross-bridges that interact with $r$-th binding site will have the same kinetic properties. If the number of cross-bridges in each group is large enough, then its stochastic description can be replaced with deterministic one. To describe each group, we used corresponding kinetic equations.

Assuming that unbound cross-bridges can be attached only to the closest binding sites on the left $(r=-1)$ or on the right $(r=1)$ and taking into account scheme (1), the set of kinetic equations that determine the dynamics of fraction of the bound cross-bridges with the distance to the closest binding site on the right $\Delta^{k}$ is as follows:

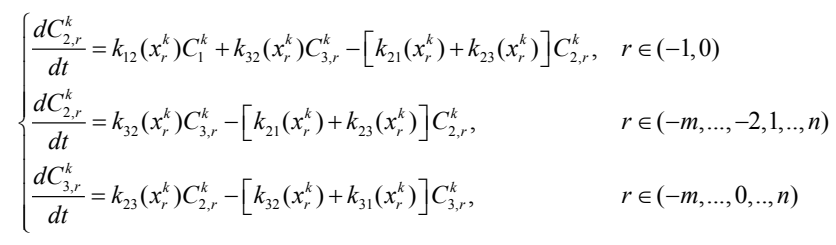

where $C_{i, r}^{k} \equiv C_{i, r}^{k}\left(x_{r}^{k}, t\right)$ is fraction of bound cross-bridges in $i$-th state which interact with $r$-th binding site located at distance $x_{r}^{k}$ at the moment $t ; C^{k}{ }_{1} \equiv C^{k}{ }_{1}\left(\Delta^{k}, t\right)$ is fraction of unbound cross-bridges that have distance $x^{k}{ }_{1}=\Delta^{k}$ to the closest binding site on the right at the moment $t$. We denote the set of all $C_{i, r}^{k}$ as $C_{i}=\left\{C^{k}{ }_{i, r} \mid k=1 \ldots M, \mathrm{r}=-m, \ldots, n\right\}$. Normalizing conditions should take into account the fact that bound cross-bridges with $x^{k}{ }_{r}=\Delta^{k}+r l_{a n}, \mathrm{r} \in(-m, \ldots$, $n$ ) after unbounding will join the group of unbound crossbridges with the distance to the closest binding site on the right $\Delta^{k}$. Normalizing conditions appear as follows:

$$
C_{1}^{k}+\sum_{i=2}^{3} \sum_{r=-m}^{n} C_{i, r}^{k}=1
$$

Distance $\Delta^{k} \in\left[0 ; l_{a n}\right)$ is a circular value, and reaching its upper bound, it moves to the lower and vice versa. The distance $x_{r}^{k} \in\left[r l_{a n} ;(r+1) l_{a n}\right)$ will be transformed into $x^{k}{ }_{r-1}$ at reaching its lower bound and into $x_{r+1}^{k}$ when it reaches its upper bound. In accordance with this, the following boundary conditions should be imposed on the fractions of bound cross-bridges:

$$
\begin{aligned}
& C_{i, r}^{k}\left((r+1) l_{a n}\right)=C_{i, r+1}^{k}\left((r+1) l_{a n}\right), \quad i=2,3, \\
& r \in(-m+1, \ldots, n-1) \\
& C_{i,-m}^{k}\left(-m l_{a n}\right)=0, C_{i, n}^{k}\left((n+1) l_{a n}\right)=0 \quad i=2,3
\end{aligned}
$$

The system of equations (7) must be completed by adding mechanical equations that define the time evolution of thin filament position $y_{a n}$. In isotonic condition if the force of inertia is disregarded this equation will be the following:

$\beta_{a n} \frac{d y_{a n}}{d t}=\left(F_{c b}+F_{e x t}\right) N$

where $\beta_{a n}$ is coefficient of viscous friction, $F_{c b}$ is an average force of all bound cross-bridges, $F_{\text {ext }}$ is external force, both forces are per cross-bridge. Force $F_{c b}$ is calculated as:

$F_{c b}=-\frac{k_{c b}}{N} \sum_{k=1}^{M} \sum_{i=2}^{3} \sum_{r=-m}^{n} p_{k} \xi_{i, r}^{k} C_{i, r}^{k}$

where $p_{k}$ represents the number of cross-bridges of the single pair of thick-thin filament with the distance to the closest binding site of $\Delta^{k}$. In case $N \leq T_{c b}$ all $N$ of cross-bridges of the single pair of contractile filaments have different $\Delta^{k}$ then $M=N$ and all $p_{k}=1$. If $N>T_{c b}$, then some of cross-bridges from the pair will have the same value of $\Delta^{k}$, in this case $p^{k}$ will be equal to the number of cross-bridges that have the distance to the closest binding site on the right $\Delta^{k}$ and $M=T_{c b}$.

Computational algorithm for numerical solution of the system of equations (7), (8) and (10) with boundary conditions (9) is given in Supplementary Methods, Section 1.

It is also possible to add noise into obtained deterministic ODE model. In the Monte Carlo simulation noise is related to stochasticity of chemical transitions. To add similar noise to deterministic ODE model we can replace ODE (Eq. 7), which has a general form of $d C_{i, r}^{k} / d t=f\left(C_{i, r}^{k}\right)$, with appropriate stochastic differential equations:

$d C_{i, r}^{k}=f\left(C_{i, r}^{k}\right) d t+g d W$

where $g$ is diffusion coefficient, $W$ is Wiener process.

\section{Approximation of solutions of Huxley-based model}

Suggested ODE model also can be applied for approximation of numerical solutions of PDE models based on Huxley's formalism, with normalizing conditions that correspond to Eq. 8. According to this formalism the distance from cross-bridge to the closest binding site is a continuous 
Tale 1. Simulation parameters

\begin{tabular}{lc}
\hline Parameter & Value (unit) \\
\hline$d_{1}$ & $0(\mathrm{~nm})$ \\
$d_{2}$ & $8(\mathrm{~nm})$ \\
$k_{c b}$ & $2(\mathrm{pN} / \mathrm{nm})$ \\
$k_{12}(x)$ & $10^{3} \exp \left(0.1 k_{c b} x^{2} / k_{B} T\right)\left(\mathrm{s}^{-1}\right)$ \\
$k_{23}(x)$ & $10\left(\mathrm{~s}^{-1}\right), \quad x>-0.1(\mathrm{~nm})$ \\
& $10^{4}\left(\mathrm{~s}^{-1}\right), \quad x \leq-0.1(\mathrm{~nm})$ \\
$k_{31}(x)$ & $10^{3}\left(\mathrm{~s}^{-1}\right), \quad x>-2.1(\mathrm{~nm})$ \\
$k_{13}(x)$ & $10^{4}\left(\mathrm{~s}^{-1}\right), \quad x \leq-2.1(\mathrm{~nm})$ \\
$G_{1}^{0}$ & 0 \\
$G_{2}$ & $0\left(\mathrm{k}_{\mathrm{B}} \mathrm{T}\right)$ \\
$G_{3}$ & $-4.3\left(\mathrm{k}_{\mathrm{B}} \mathrm{T}\right)$ \\
$\beta_{a n}$ & $-18.7\left(\mathrm{k}_{\mathrm{B}} \mathrm{T}\right)$ \\
\hline
\end{tabular}

quantity. If in contractile system with discrete lattice the size of a discrete set $\Delta$ converge to infinity, then the distances between myosin heads and binding sites becomes a continuous quantity and such contractile system can be described with PDE model. In the case of the above considered contractile system composed from numerous pairs of thick-thin filaments (Fig. 4), this situation can be reproduced by introducing mutual axial offsets from interval $\left(0, l_{a n}\right]$ to different thin filaments. Assume that as results of such modification we have set $\Delta$ which consists of the following $K$ values:

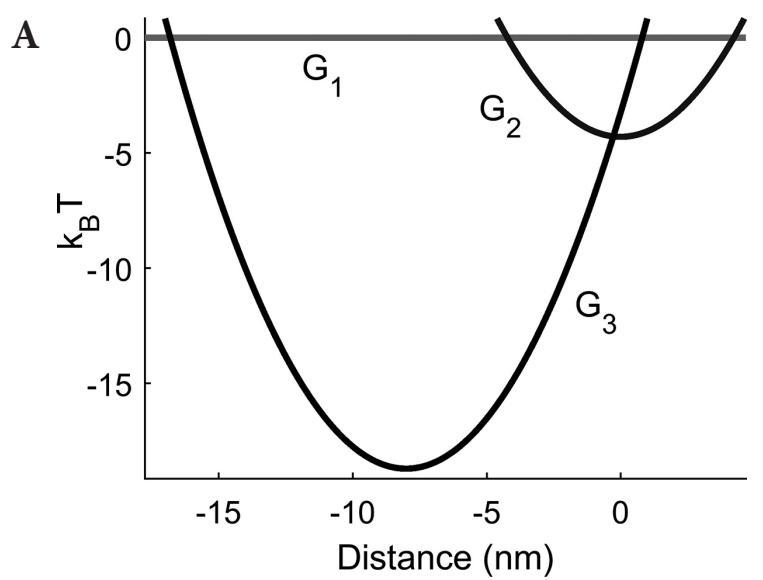

$\Delta^{k}=(k-1) \frac{l_{a n}}{K}, \quad k=1, \ldots, K$

According to Huxley's approach (Huxley 1957) when $K$ goes to infinity such contractile system can be described by system of PDEs. The system of this equations together with normalizing conditions correspond to those of the discrete lattice model (Eq. 8) are given in Supplementary Methods, Section 2.

\section{Simulation parameters}

Further we consider the parameters of simulation that are common for all models (see also Table 1). Mechanochemical cross-bridge cycle includes three states (scheme 1). It is assumed that the first bound state (state 2 on scheme 1 ) is weakly bound, transition $1 \rightarrow 2$ is not accompanied by cross-bridge conformational changes $\left(d_{1}=0 \mathrm{~nm}\right)$. The second state is strongly attached force-generating state, transition $2 \rightarrow 3$ is followed by power stroke of $d_{2}=8 \mathrm{~nm}$ size. Dependencies of forward transition rates on distance $x$ are given in Table 1 and Fig. 5. Revers transition rates were found from the ratio $k_{j i}=k_{i j} \exp \left(G_{j}-G i\right)$, where $G_{i}(x)=k_{c b}\left(x+\sum^{i-1} k=1 d_{k}\right)^{2} / 2+\mathrm{G}^{0}{ }_{\mathrm{i}}$ is dependence of free energy of state $i$ on distance $x$. In these equations energies $G_{i}$ and $G_{i}{ }^{0}$ are in units of $k_{B} T$ where $k_{B}$ is Boltzmann constant and $T=300 \mathrm{~K}$ is the absolute temperature. The selected parameters are intended to illustrate general principle, and the results similar to calculated below can be also obtained under their different values. An essential issue is dependence of the rate of transition $2 \rightarrow 3$ on distortion, when distortion decreases $k_{23}(x)$ should increase non-linearly. Such dependence can be found in many other models.

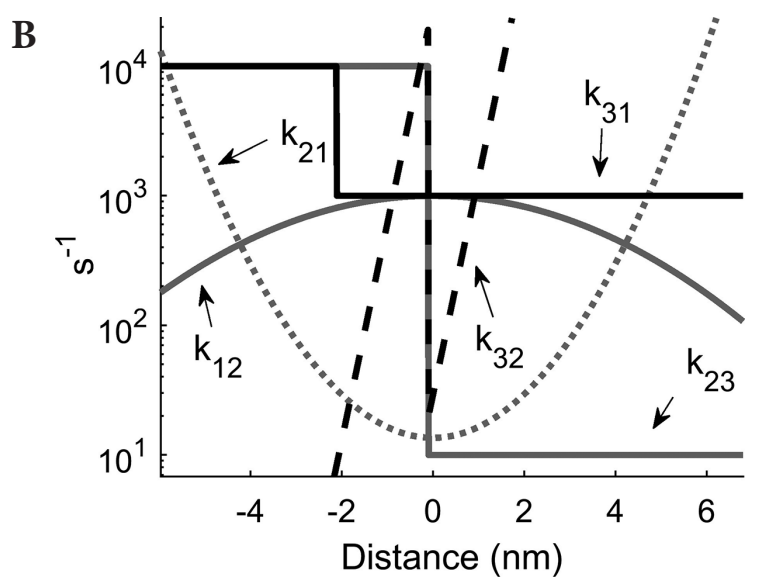

Figure 5. Free energy profiles (A) and transition rates (B) are plotted against distance $x$ which represents relative distance between cross-bridge and binding site. 


\section{Computational implementation}

Model with Monte Carlo simulation and ODE model was implemented in $\mathrm{C}++$ as console application. ODE and SDE models also have been implemented in MATLAB/GNU Octave package. For numerical solving of ODEs and SDEs systems, libraries SUNDIALS (Hindmarsh et al. 2005), SDETools (github.com/horchler/SDETools) and standard MATLAB/GNU Octave ODE solvers were used. ODE and SDE solvers from above-mentioned libraries support event detection, this feature is essential for boundary conditions realization (Eq. 9).

\section{Results}

\section{Isotonic transient response to a force step}

In order to study the effects of contractile apparatus structure on dynamics of cross-bridge ensemble, isotonic transient response at different model parameters was simulated. The simulation was carried out both within deterministic ODE model and, for comparison, with spatially explicit model that uses Monte Carlo simulation. For simulation of isotonic transient response, contractile system was previously activated isometrically after that it was allowed to contract isotonically against different external loads. Values of external force in isotonic mode were less than isometric force.

At first we consider the lattice with simpler geometry. We assume that $l_{m n}=43.2 \mathrm{~nm}$ and $l_{a n}=35.1 \mathrm{~nm}$, the number of cross bridges $N=13$. For these parameters, according to Eq. 2 and 3, $T_{c b}=13$ and $s=2.7 \mathrm{~nm}$. Because $N$ is equal to $T_{c b}$, values $\Delta^{k}$ will be uniformly distributed among all cross-bridges and $\Delta^{k+1}-\Delta^{k}=s$. Fig. 6 shows trajectories for isotonic contraction. Trajectories are stepwise and complete steps size (height of the plateau plus the jump height) are equal to $2.7 \mathrm{~nm}$. As it is observed from Fig. 6A, Monte Carlo simulation of contractile system composed of large number of identical pairs of thin-thick filaments and its deterministic ODE approximation fairly coincide. The velocity of contraction increases at external force $F_{\text {ext }}$ decreasing due to reducing of pauses between steps (Fig. 6B). At the intermediate velocities of contraction, single steps alternate with double ones (Fig. 6B, $F_{\text {ext }}=0.18 \mathrm{pN}$ per cross-bridge).

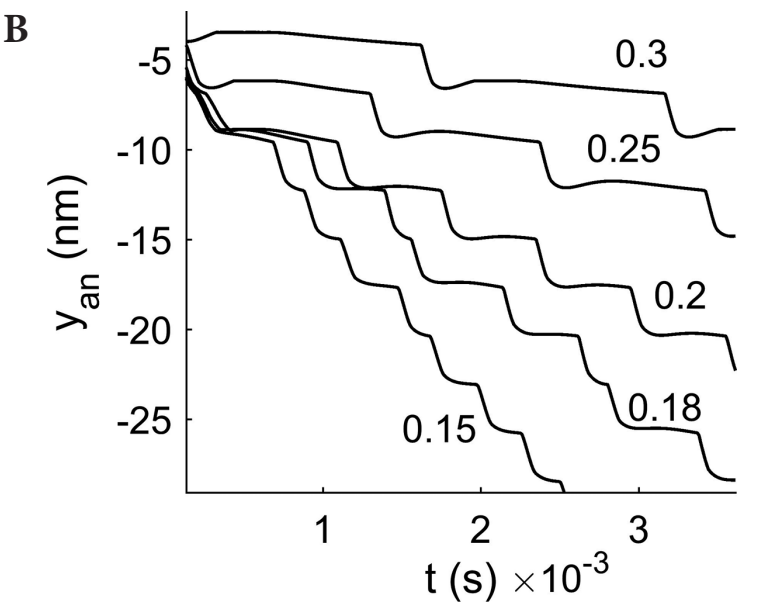

Figure 6. Isotonic transient responses received in spatially explicit model with Monte Carlo simulation and in its deterministic ODE approximation, with lattice parameters $l_{m n}=43.2 \mathrm{~nm}$ and $l_{a n}=35.1 \mathrm{~nm}$. A. Comparison of Monte Carlo and deterministic simulations (curves are marked as MC and ODE respectively) at external isotonic force $F_{\text {ext }}=0.4 \mathrm{pN}$ per cross-bridge. In Monte Carlo simulation, the number of thin-thick filament pairs connected in parallel was $1.5 \times 10^{3}, N=13$. B., C. Simulation results of ODE model at various values of external loads (curves are marked by values of $F_{\text {ext }}$ in $\mathrm{pN}$ per cross-bridge). The results on the figures $\mathrm{B}$ and $\mathrm{C}$ were calculated with $N=13$ and $N=20$ respectively. 
A

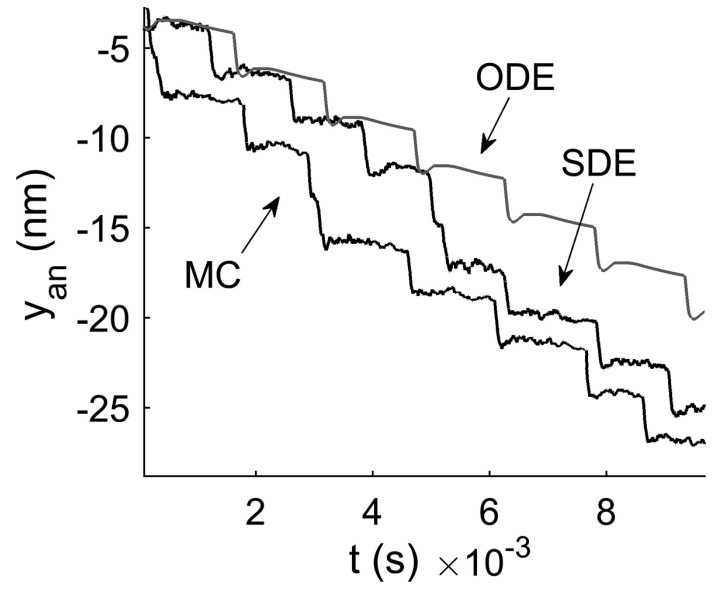

B

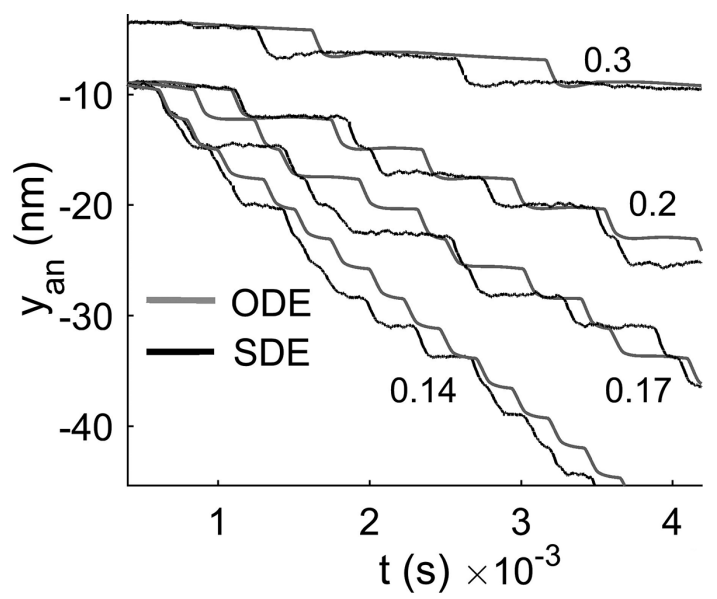

Figure 7. Effect of noise on contractile dynamics. Isotonic transient responses retrieved in SDE model, Monte Carlo simulation and in its deterministic approximation with lattice parameters: $l_{m n}=43.2 \mathrm{~nm}$, and $l_{a n}=35.1 \mathrm{~nm}, N=13$ are shown. A. Comparison of the results of the SDE model and Monte Carlo simulation (curves are marked as SDE and MC respectively). Also, simulation result of ODE model is shown (curve is marked as ODE). In all cases, external isotonic force was $F_{\text {ext }}=0.4 \mathrm{pN}$ per cross-bridge. In Monte Carlo simulation, the number of thin-thick filament pairs connected in parallel was 150, and in SDE model $g=0.6$. The arrows show steps of $5.4 \mathrm{~nm}$. B. Comparison of the results of the SDE and ODE models (curves are marked as SDE and ODE respectively) at various values of external loads (curves are labeled by values of $F_{\text {ext }}$ in $\mathrm{pN}$ per cross-bridge). In SDE model $g=0.4$.

Double steps have size of $5.4 \mathrm{~nm}$ and emerge as the result of single $2.7 \mathrm{~nm}$ steps fusion. The pauses between such steps are almost absent.

If the number of cross-bridges is increased to $N=20$, some of them will have identical $\Delta^{k}$. In this case under all external loads in trajectories of ODE model there are steps of $2.7 \mathrm{~nm}$ and $5.4 \mathrm{~nm}$ size. Their sequence forms a certain pattern (Fig. 6C).

To study the influence of stochasticity on dynamics of contractile system the ODEs (Eq. 7) in deterministic model was replaced with SDEs (Eq. 12). Trajectories of SDE model reproduce fairly good results of the Monte Carlo simula-

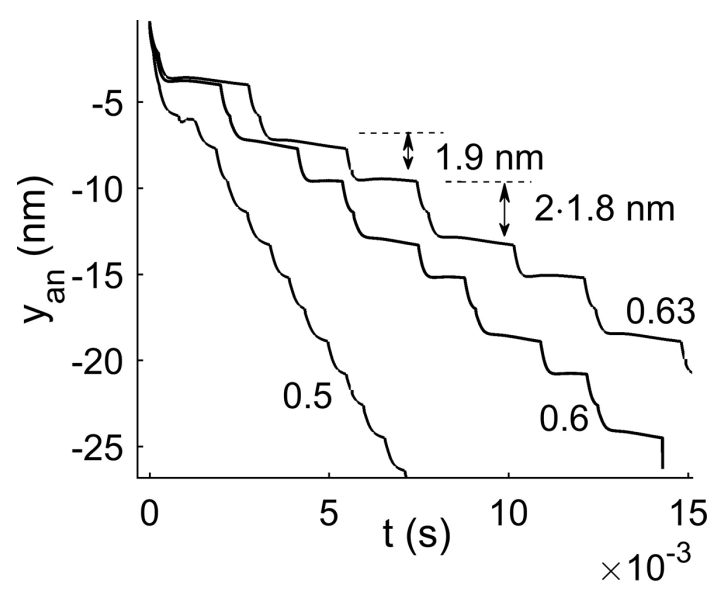

Figure 8. Isotonic transient responses retrieved in ODE model with lattice parameters $l_{m n}=42.9 \mathrm{~nm}, l_{a n}=37.3 \mathrm{~nm}$ and $N=20$. tion even though the structure of noise is different in both models (Fig. 7A). Noise leads to formation of the fused steps with integer multiples of $2.7 \mathrm{~nm}$ (Fig. 7A,B). In contrast to ODE model with the same parameters of the lattice, these fused steps appear under different external forces and their appearance is random. Fused steps occur more frequently and have greater sizes under lower external forces (Fig. 7B).

If as periods $l_{m n}$ and $l_{a n}$ experimental values of $42.9 \mathrm{~nm}$ and $37.3 \mathrm{~nm}$ respectively are selected, then $T_{c b}=373$ and $s=0.1 \mathrm{~nm}$. In case $N<T_{c b}$ distances $\Delta^{k+1}-\Delta^{k}$ can take different values for different $k$, but they all will be integer multiples of $0.1 \mathrm{~nm}$. The exact set of values depends on the number of cross-bridges $N$ as well as on mutual displacement of thick and thin filament. For example, at $N=20$ value set $\Delta^{k+1}-\Delta^{k}$ will mainly consist of $1.8 \mathrm{~nm}$ and $1.9 \mathrm{~nm}$ values. In the ODE model, trajectory steps will also have sizes equal either to those values or their sum (Fig. 8).

\section{Mechanism of stepwise motion}

To analyze the mechanism of stepwise contraction, the dynamics of discrete distributions $C_{i}(t, x)$ was researched (Fig. 9). Under contraction, the distributions $C_{i}$ move to the left along the $x$-axis (Fig. 9A-F) and their form changes simultaneously.

\section{The pauses between jumps}

Drift on the plateau between consecutive steps (Fig. 9A-C) takes place due to increasing of weakly bound cross-bridges 
fraction of the group 3 (Fig. 9A-C, fractions of weakly and strongly bound cross-bridges of this group are marked with arrow and number " 3 ") caused by the chemical transitions $1 \rightarrow 2$. At the beginning of a plateau this process is compensated by unbinding of strongly bound cross-bridges of the group 3 , and as a result actin remains almost fixed (Fig. 9A, B).

\section{Jumps}

A jump in the trajectory is a result of synchronization of power strokes (transitions $2 \rightarrow 3$ ) between weakly bound crossbridges one of the discrete groups (Fig. 9C-E). The drift on the plateau leads to decreasing of the distance between weakly bound cross-bridges of the group 2 and binding sites they interact with. At a certain moment of time this distance gets less than $-0.1 \mathrm{~nm}$ and these results in sharp increasing of transition rate $2 \rightarrow 3$ (Fig. 5B). As this transition is accompanied by power stroke it also leads to dramatic rise of force $F_{c b}$ and the velocity of contraction.

\section{Size of elementary steps}

When trajectory attains a new plateau, distributions $C_{i}$ take the same form as at the beginning of the cycle, and the process repeats itself. Thus, one cycle of distribution changes continues until each of the cross-bridges groups takes the position occupied by the neighboring group at the beginning of the cycle (which is as far as $s=2.7 \mathrm{~nm}$ ). For example, group 2 at the end of the cycle will occupy the position of group 3 which the latter have previously occupied at the beginning of the cycle. Therefore, total step amplitude is related to spatial duration of cyclic changes. Value of the power stroke does not directly influence the step size.

\section{Formation of the double steps in ODE model}

If external force is not large, the group of cross-bridges that forms a jump in trajectory can also initiate synchronous performance of power-strokes for its adjacent group, it leads to formation of the double steps (Fig. $6 \mathrm{~B}, F_{\text {ext }}=0.18 \mathrm{pN}$ per cross-bridge). For example, if while performing a jump, group 2 advances the thin filament, so that at the end of this jump (Fig. 9E) group 1 is located behind the distance $x=-0.1 \mathrm{~nm}$, this will result in formation of instant second jump, avoiding the phase of the plateau. Fusion is limited by two steps because group 1 will advance thin filament to some smaller distance than group 2. Because second jump is not preceded with pause, then group 1 will have less time in the region $x>-0.1 \mathrm{~nm}$ and consequently will have less amount of weakly bound cross-bridges at the beginning of this jump. If parameters of the model are chosen so that the distances $\Delta^{k+1}-\Delta^{k}$ are sufficiently small, then fusion of more than two steps can occur in the same manner.

\section{Formation of fused steps in SDE model}

Mechanism of the steps fusion under noise condition is similar to that in the ODE model but throwing of another group, which will perform the next jump, over the boundary $x=-0.1 \mathrm{~nm}$ will be carried out due to random instantaneous increase in force $F_{c b}$. Moreover, at external force decreasing, relative distance from this group to boundary $x=-0.1 \mathrm{~nm}$ at the beginning of the new step will be reducing (in Fig. $9 \mathrm{~F}$

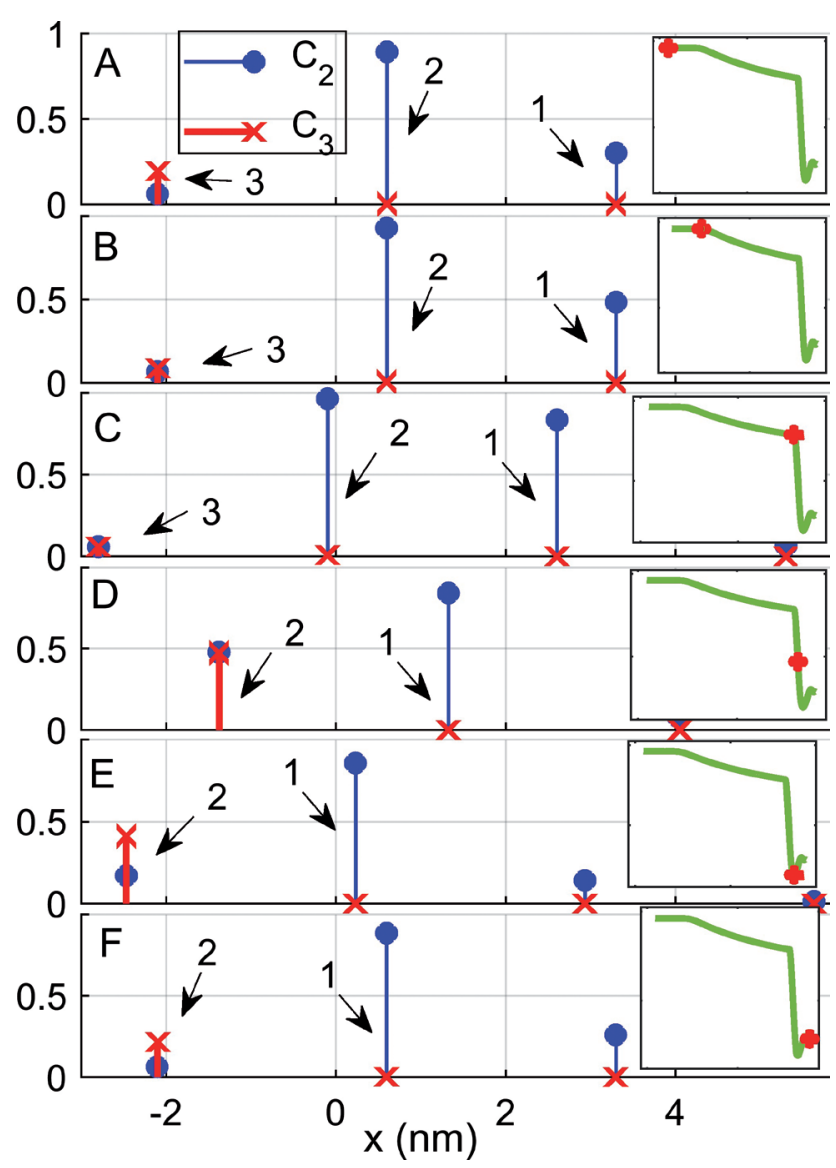

Figure 9. Mechanism of stepwise contraction. A.-F. Dynamics of discrete distributions $C_{i}(t, x)$ of bound cross-bridges when contractile system performs one of the steps in trajectory of length change. Distributions shown in A-F correspond to different moments of time. Vertical lines with markers at the top represent different values of $C_{i, r}^{k}\left(x_{r}^{k}, t\right)\left(C_{i, r}^{2}\right.$ and $C_{i, r}^{3}$ marked with $\bullet$ and $\times$ respectively $)$. A vertical line which represent value $C^{k}$ i,r is located on the $x$-axis at the point $x^{k}$. The distance between any adjacent lines along the $x$-axis is equal to $s=2.7 \mathrm{~nm}$. The lines for some of $C_{i, r}^{k}$ are marked by arrows with numbers. The fragment of the trajectory of the ODE model from Fig. 6A is shown on the inserts. Marker on the trajectory indicates current actin position. The results were calculated in ODE model at $l_{m n}=43.2 \mathrm{~nm}, l_{a n}=35.1 \mathrm{~nm}, N=13$ and $F_{\text {ext }}=0.4$ pN per cross-bridge. 

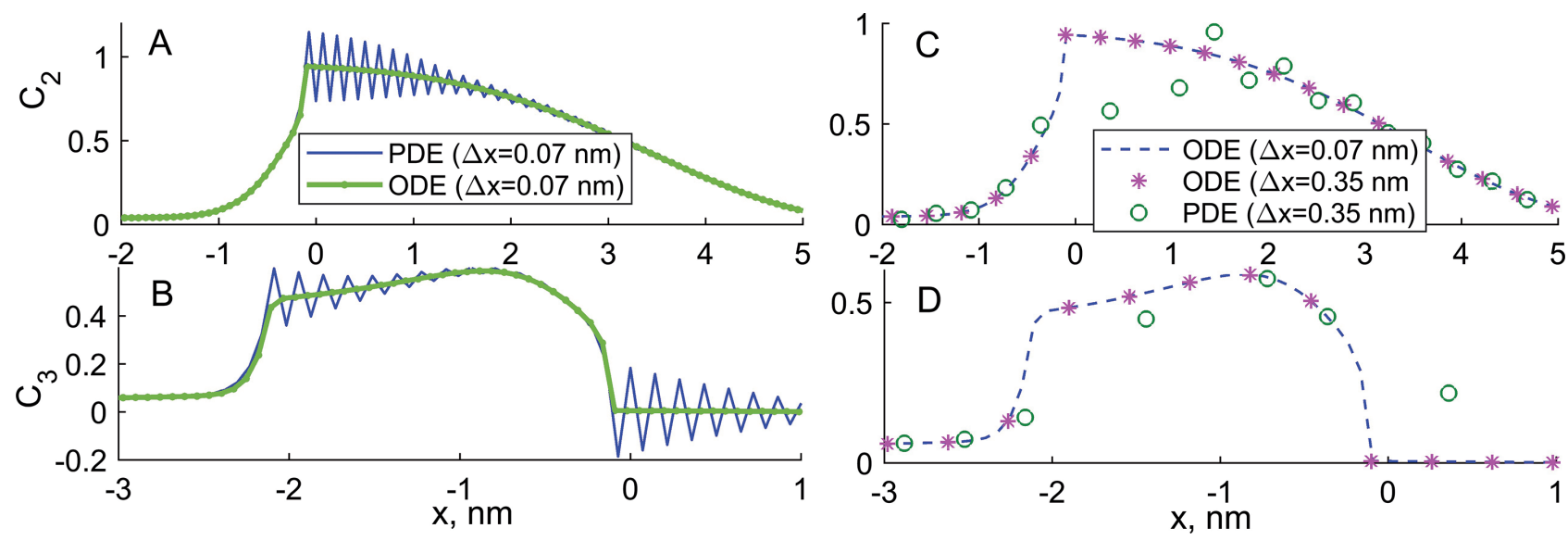

Figure 10. Distributions $C_{i}(x)$ of bound cross-bridges at isokinetic contraction obtained as numerical solution of ODE model (curves are marked as "ODE") and PDE model (curves are marked as "PDE") at different values of step $\Delta x$.

this distance is between group 1 and point $x=-0.1 \mathrm{~nm}$ ) so fusion probability will increase with force $F_{\text {ext }}$ decreasing.

The above given analysis shows that stepwise dynamics observed in the model is a result of cooperative effects related to structural constraints of discrete lattice. At contraction, steps occur due to synchronization of power strokes between cross-bridges of one of the discrete groups. Another essential condition for synchronization is nonlinearity of strain dependent rate constants of the transitions that are related with significant changes of cross-bridge strain (power stroke generation) or contractile system stiffness (attachment or detachment of cross-bridges). In the above-mentioned simulations such rate constant is $k_{23}(x)$. It characterizes the rate of the power stroke transition, this rate sharply increases with decreasing $x$ after $x=-0.1 \mathrm{~nm}$ (Fig. 5B).

We have considered the simulation of isometric contraction but stepwise sliding can also be received under the stretching of contractile system. In this case jumps will be resulted from reverse power strokes synchronization which is related to transitions $3 \rightarrow 2$. This synchronization will arise due to nonlinear rate increasing of reverse power stroke $\left(k_{32}(x>0)\right)$ under increasing of $x$ (Fig. 5B).

\section{Approximation solutions of PDE model}

As stated above, ODE model (Eqs. 7-10) can also be used to approximate the solutions of PDE models based on Huxley formalism. We compared the results of simulation of isokinetic contraction in ODE model and corresponding PDE model derived within the Huxley approach (see Supplementary Eqs. S3 and S4). In ODE model the set of $\Delta^{k}$ values is formed based on Eq. 13 and as a result all values $x_{r}{ }_{r}$ are uniformly distributed along the interval of integration with step $\Delta x=l_{a n} / K$.
For numerical solution of PDEs (Supplementary Eq. S3), its spatial coordinate $x$ is discretized via $K(m+n)+1$ points and $K(m+n)$ intervals of length $\Delta x=l_{a n} / K(m+n)$ each. Spatial derivatives in the right side PDEs (Supplementary Eq. S3) are approximated by central differences. For numerical solving of the obtained system of the ODEs we used the same numerical methods (the same solvers of MATLAB/Octave package) as for ODE model (Eq. 7). Likewise the simulation from previous section, cross-bridge cycle is composed of three states, with parameters from Table 1. Isokinetic contraction has preceded isometric activation in the simulation. The velocity of contraction was $1 \mu \mathrm{m} / \mathrm{s}$.

The results of simulation are shown on Fig. 10. As it can be observed numerical solutions of PDE model are unstable, they have significant oscillations. Without those oscillations, PDE and ODE model solutions match closely (Fig. 10A,B). Under decreasing of $\Delta x$, those oscillations decrease and solutions of PDE model approach to those of ODE model. In contrast the solutions of ODE model remain stable and their form doesn't change even with large $\Delta x$ (or small $K$ ) (Fig. 10C,D).

\section{Discussion}

Results of the simulation of the isotonic transient show that the structure of contractile system can play significant role in its functioning. In particular, in the model of contractile system composed of the pair of filaments can occur cooperativity between molecular motors due to their interactions within discrete filaments lattice, this cooperativity leads to stepwise motion of the thin filament. Can we consider oscillations observed in the model similar to the experimental ones? Similarly to the experimental results (Pollack et al. 
2005), the model allows to obtain steps with height which is integer multiple of some elementary step size. Also the pauses duration between steps and relative probability of appearance large and small steps shows a dependency on the sliding velocity similar to experimental. However, the model faces difficulties with obtaining experimental value for elementary step size of $2.7 \mathrm{~nm}$.

In the model the constraints on the size of the elementary steps are imposed by values $\Delta^{k+1}-\Delta^{k}$. Their sizes are equal to or a combination of $\Delta^{k+1}-\Delta^{k}$. The set of $\Delta^{k+1}-\Delta^{k}$, in turn, depends on geometry of filaments lattice (periods $l_{m n}$ and $l_{a n}$ ), as well as on the number of cross-bridges $N$. Moreover, the exact size of the steps, which will be drawn from the available values $\Delta^{k+1}-\Delta^{k}$, depends on functional form of transition rates in cross-bridge cycle.

As we have observed experimental value for elementary step size can be obtained in the model at periods $l_{m n}$ and $l_{a n}$ that are somewhat different from experimental ones. Moreover, since dependence of elementary step size on parameters $l_{m n}$ and $l_{a n}$ has complex nonlinear character, the model is very sensitive to their changes. On this background we can conclude that it is unlikely to explain the experimentally observed length oscillations only on the ground of cooperative effects in myosin cross-bridges related to restrictions imposed by discrete filament lattice on myosin and actin interaction.

Possible solution to overcome this problem is consideration of alternative or additional assumptions concerning the structure of the contractile system, and myosin and actin interaction. For example, existence of more complicated geometry can be assumed, when single actin filament interacts with several rows of cross-bridges of single thick filament (Suzuki et al. 2011). Else when myosin can interact not only with one actin monomer but also with a number of neighbouring ones located in the target zone (Steffen et al. 2001; Tregear et al. 2004). In the last case it will result in the distances $\Delta^{k+1}-\Delta^{k}$ that are equal or integer times smaller than the linear repeat of actin monomers along the thin filament $(2.7 \mathrm{~nm})$. Another assumption may relate to the mechanism of force generation by single cross-bridges. Based on single molecule measurement, it was assumed (Kitamura et al. 1999; Yanagida et al. 2012) that sliding of actin past myosin can be driven by biased Brownian movement of the bound myosin heads along actin filament rather than by lever arm rotation. A single myosin head moves in stepwise manner with unitary steps of $\sim 5.5 \mathrm{~nm}$. It may take $2-5$ fast steps per one hydrolyzed ATP (Kitamura et al. 1999).

\section{Conclusions}

An innovative method for deterministic ODE approximation of stochastic spatially explicit model of single pair of rigid thick and thin filaments is introduced in this study. Application of such ODE model allows to avoid extensive calculations associated with the use of Monte Carlo simulation of the spatially explicit model. ODE model retains information about constraints imposed on actin-myosin interactions caused by their arrangement in discrete filament lattice.

Simulation of isotonic length response to a force step within both models shows that the results of ODE model coincide with Monte Carlo simulation. ODE model reproduces collective effects of cross-bridge ensemble that are presented in spatially explicit model and are related to structural constraints of discrete lattice. Effects of noise associated with stochastic kinetic transitions in Monte Carlo simulation can be reproduced in approximation by replacing ODEs with SDEs system.

Within ODE model, the mechanism of stepwise motion under isotonic response is analyzed in great detail; interrelation between step size and geometrical parameters of filament lattice is studied. Discreetness of spacing between cross-bridges and binding sites and nonlinearity of strain dependent transition rates between weakly and strongly bound states are basic preconditions for stepwise motion. Despite the fact that this dynamics is qualitatively similar to those observed experimentally, the given analysis implies that the dynamics observed in experiment cannot be explained only via cooperative effects related to structural constraints of discrete lattice. Using current assumptions, the model faces principal difficulties in obtaining the step size value that is equal to experimental. Possible solution is consideration of more complicated structure of contractile system, other assumptions about the mechanism of force generation by single cross-bridge and its interaction with actin.

The suggested approach can also be applied for approximation of solutions of PDE models based on Huxley formalism. Simulation of isokinetic contraction both in ODE and PDE model respectively has shown that the results of both models coincide under sufficiently small values of discrete step in space $\Delta x$. Stability of PDE model solutions, obtained by the method of lines, is greatly dependent on the value $\Delta x$. Whereas the solutions of ODE model remain stable in spite of value $\Delta x$.

\section{References}

Campbell KS (2006): Filament compliance effects can explain tension overshoots during force development. Biophys. J. 91, 4102-4109 https://doi.org/10.1529/biophysj.106.087312

Daniel TL, Trimble AC, Chase PB (1998): Compliant realignment of binding sites in muscle: transient behavior and mechanical tuning. Biophys. J. 74, 1611-1621

https://doi.org/10.1016/S0006-3495(98)77875-0 
Duke TA (1999): Molecular model of muscle contraction. Proc. Natl. Acad. Sci. USA 96, 2770-2775 https://doi.org/10.1073/pnas.96.6.2770

Edman KA, Curtin NA (2001): Synchronous oscillations of length and stiffness during loaded shortening of frog muscle fibres. J. Physiol. 534, 553-563 https://doi.org/10.1111/j.1469-7793.2001.t01-2-00553.x

Hindmarsh AC, Brown PN, Grant KE, Lee SL, Serban R, Shumaker DE, Woodward CS (2005): SUNDIALS: Suite of nonlinear and differential/algebraic equation solvers. ACM Transactions on Mathematical Software (TOMS) 31, 363-396 https://doi.org/10.1145/1089014.1089020

Huxley AF (1957): Muscle structure and theories of contraction. Prog. Biophys. Chem. 7, 255-318

Iwamoto H (2011): Structure, function and evolution of insect flight muscle. Biophysics 7, 21-28 https://doi.org/10.2142/biophysics.7.21

Iwamoto H, Inoue K, Yagi N (2006): Evolution of long-range myofibrillar crystallinity in insect flight muscle as examined by X-ray cryomicrodiffraction. Proc. Biol. Sci. 273, 677-685 https://doi.org/10.1098/rspb.2005.3389

Kitamura K, Tokunaga M, Iwane AH, Yanagida T (1999): A single myosin head moves along an actin filament with regular steps of 5.3 nanometres. Nature 397, 129-134 https://doi.org/10.1038/16403

Liu X, Pollack GH (2004): Stepwise sliding of single actin and Myosin filaments. Biophys. J. 86, 353-358 https://doi.org/10.1016/S0006-3495(04)74111-9

Nagornyak EM, Pollack GH, Blyakhman FA (2004): Step size in activated rabbit sarcomers is ndependent of filament overlap. J. Mech. Med. Biol. 4, 485-498 https://doi.org/10.1142/S0219519404001181

Placais PY, Balland M, Guerin T, Joanny JF, Martin P (2009): Spontaneous oscillations of a minimal actomyosin system under elastic loading. Phys. Rev. Lett. 103, 158102 https://doi.org/10.1103/PhysRevLett.103.158102

Pollack GH, Blyakhman FA, Liu X, Nagomyak E (2005): Sarcomere dynamics, stepwise shortening and the nature of contraction. In: Sliding Filament Mechanism in Muscle Contraction: Fifty Years of Research. (Ed. H Sugi), pp. 113-126, Springer, Boston https://doi.org/10.1007/0-387-24990-7_9

Smith DA, Mijailovich SM (2008): Toward a unified theory of muscle contraction. II: predictions with the mean-field approximation. Ann. Biomed. Eng. 36, 1353-1371 https://doi.org/10.1007/s10439-008-9514-z
Squire JM, Al-Khayat HA, Knupp C, Luther PK (2005): Molecular architecture in muscle contractile assemblies. Adv. Protein Chem. 71, 17-87 https://doi.org/10.1016/S0065-3233(04)71002-5

Squire JM, Knupp C, Luther PK (2008): Zebrafish-topical, transparent, and tractable for ultrastructural studies. J. Gen. Physiol. 131, 439-443 https://doi.org/10.1085/jgp.200810015

Steffen W, Smith D, Simmons R, Sleep J (2001): Mapping the actin filament with myosin. Proc. Natl. Acad. Sci. USA 98, 14949-14954 https://doi.org/10.1073/pnas.261560698

Suzuki M, Fujita H, Ishiwata S (2005): A new muscle contractile system composed of a thick filament lattice and a single actin filament. Biophys. J. 89, 321-328 https://doi.org/10.1529/biophysj.104.054957

Suzuki M, Ishiwata S (2011): Quasiperiodic distribution of rigor cross-bridges along a reconstituted thin filament in a skeletal myofibril. Biophys. J. 101, 2740-2748 https://doi.org/10.1016/j.bpj.2011.10.040

Tanner BC, Daniel TL, Regnier M (2007): Sarcomere lattice geometry influences cooperative myosin binding in muscle. PLoS Comput. Biol. 3, 1195-1211 https://doi.org/10.1371/journal.pcbi.0030115

Tregear RT, Reedy MC, Goldman YE, Taylor KA, Winkler H, Franzini-Armstrong C, Sasaki H, Lucaveche C, Reedy MK (2004): Cross-bridge number, position, and angle in target zones of cryofixed isometrically active insect flight muscle. Biophys. J. 86, 3009-3019 https://doi.org/10.1016/S0006-3495(04)74350-7

Vilfan A, Duke T (2003): Instabilities in the transient response of muscle. Biophys. J. 85, 818-827 https://doi.org/10.1016/S0006-3495(03)74522-6

Yakovenko O, Blyakhman F, Pollack GH (2002): Fundamental step size in single cardiac and skeletal sarcomeres. Am. J. Physiol. Cell Physiol. 283, C735-742 https://doi.org/10.1152/ajpcell.00069.2002

Yanagida T, Ishii Y (2012): Muscle contraction mechanism based on single molecule measurements. J. Muscle Res. Cell Motil. 33, 395-402 https://doi.org/10.1007/s10974-012-9332-7

Received: November 8, 2017

Final version accepted: January 29, 2018

First published online: June 29, 2018 